\title{
Expression of neonatal Nav1.5 in human brain astrocytoma and its effect on proliferation, invasion and apoptosis of astrocytoma cells
}

\author{
DEGUANG XING $^{1 *}$, JUN WANG $^{1 *}$, SHAOWU OU $^{1}$, YUNJIE WANG $^{1}$,

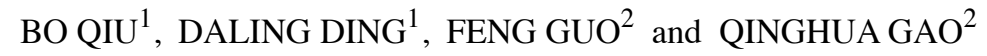 \\ ${ }^{1}$ Department of Neurosurgery, The First Hospital of China Medical University, Shenyang 110001; \\ ${ }^{2}$ Department of Pharmaceutical Toxicology, School of Pharmacy, China Medical University, Shenyang 110001, P.R. China
}

Received January 22, 2014; Accepted March 28, 2014

DOI: 10.3892/or.2014.3143

\begin{abstract}
In the present study, we designed and conducted a series of assays to determine the expression of voltage-gated sodium channel (VGSC) neonatal isoform Nav1.5 (nNav1.5) in human brain astrocytoma and its effect on the proliferation, migration, invasion and apoptosis of astrocytoma U251 cells. The results showed that nNav1.5 mRNA and protein were expressed in both human brain astrocytoma and normal brain tissues, but their expression levels in astrocytoma were significantly higher $(\mathrm{P}<0.05)$. In astrocytomas, nNav1.5 mRNA and protein levels were also different $(\mathrm{P}<0.05)$ and were correlated with pathological grades. The immunofluorescence confocal microscopy observations demonstrated that $\mathrm{nNav1.5}$ protein was expressed in the nucleus, cytoplasm and membrane of the astrocytoma cells. After transfection, the small interfering RNA (siRNA) targeted to nNav1.5 significantly reduced the expression levels of SCN5A/nNav1.5 mRNA and protein by $57.2 \%(\mathrm{P}<0.05)$ and $66.6 \%(\mathrm{P}<0.05)$, respectively. The MTT, wound healing, Matrigel invasion and flow cytometric assays confirmed that following siRNA downregulation of the expression of the SCN5A/nNav1.5 gene, the in vitro proliferation and in vitro invasiveness of the U251 cells were significantly reduced ( $\mathrm{P}<0.05$ for both comparisons), and the apoptosis rate was significantly increased $(\mathrm{P}<0.05)$. These results revealed that nNav1.5 expression in human brain astrocytoma was upregulated, and its expression was positively correlated with the degree of malignancy. Additionally, reduced nNav1.5
\end{abstract}

Correspondence to: Dr Yunjie Wang, Department of Neurosurgery, The First Hospital of China Medical University, No. 155 North Nanjing Street, Heping, Shenyang 110001, P.R. China

E-mail: wyj024@vip.sina.com

*Contributed equally

Abbreviations: VGSC, voltage-gated sodium channel; nNav1.5, neonatal Nav1.5; CNS, central nervous system; RNAi, RNA interference; WHO, World Health Organization; PI, propidium iodide; DAPI, 4,6-diamidino-2-phenylindole

Key words: voltage-gated sodium channel, nNav1.5, astrocytoma, siRNA, RNA interference expression significantly suppressed the proliferation and invasiveness of astrocytoma cells, indicating a new target in the molecular diagnosis and therapy of astrocytoma.

\section{Introduction}

Malignant astrocytoma is one of the most common primary tumors of the central nervous system (CNS), accounting for $\sim 40-60 \%$ of intracranial tumors (1), and patients exhibit poor prognosis and an extremely short median survival time, which are mainly related to the biological characteristics of abnormal proliferation and invasive growth of astrocytoma cells. Previous studies have shown that various types of $\mathrm{Na}^{+}$channels are widely distributed on the astrocytoma cell membrane (2). These channels play important roles in stabilizing the membrane potential, intracellular $\mathrm{Na}^{+}$balance, and $\mathrm{Na}^{+} / \mathrm{K}^{+}$ATPase activity of astrocytoma cells as well as participating in the processes of cell cycle regulation and apoptosis, which directly and indirectly affect cell proliferation and metastasis in astrocytomas. Thus, $\mathrm{Na}^{+}$channels are expected to become a new target for gene therapy for astrocytoma (3-6). Our previous studies found that a new neonatal isoform of the Nav1.5 $\mathrm{Na}^{+}$channel, neonatal Nav1.5 (nNav1.5), was functionally expressed in human brain neuroblastoma NB-1 cells (7-9). This neonatal isoform is considered to be the re-expression of an embryonic gene or oncogene, and its expression is intimately related to the occurrence and development of tumors, which has been confirmed in lymphoma and breast cancer (10-12). To date, no study concerning the specific expression of nNav1.5 in human brain astrocytoma and its effect on the processes of tumor cell proliferation and invasion has been reported.

The present study investigated the expression of nNav1.5 in human brain astrocytoma in detail to localize nNav1.5 protein expression in astrocytoma cells for the first time. RNA interference (RNAi) technology was applied to transfect a small interfering RNA (siRNA) against nNav1.5 into U251 cells, and its effects on cell proliferation, metastasis, invasion, and apoptosis were observed to explore the relationship between expression of the voltage-gated sodium channel (VGSC) $\alpha$ subtype nNav1.5 in astrocytoma cells and the biological behavior of the tumor cells, thereby providing new ideas and methods for molecular and gene therapies for astrocytoma. 
Table I. Primers used for real-time RT-PCR.

Gene

\begin{tabular}{|c|}
\hline $\begin{array}{l}\mathrm{nNav} 1.5(\mathrm{~F}) \\
\mathrm{nNav1} 1.5(\mathrm{R})\end{array}$ \\
\hline$\beta$-actin $(F)$ \\
\hline$\beta$-actin $(\mathrm{R})$ \\
\hline
\end{tabular}

\section{5'-ACCTTGTGGTCCTGAATCTC-3' \\ 5'-GAGGCACCTTCTCCGTCT-3' \\ 5'-TCACCCACATGTGCCCATCTACGA-3' \\ 5'-CAGCGGAACCGCTCATTGCCAATGG-3'}

282

295

nNav1.5, neonatal Nav1.5.

\section{Materials and methods}

Source of specimens. The astrocytoma tissues were collected from 68 patients with brain astrocytomas admitted to the Department of Neurosurgery at the First Hospital of China Medical University from October 2011 to October 2012. The tumors were pathologically confirmed after surgery by two experienced neuropathologists, and 12 normal brain tissue samples were collected from the available normal brain tissue specimens following decompression surgery for traumatic brain injury. The 68 patients with brain astrocytomas included 36 men and 32 women with a median age of 44 years (range 23 to 67 years). According to the 2007 classification of CNS tumors by the World Health Organization (WHO), the pathological diagnosis showed 4 cases of tumors in grade I, 21 cases in grade II, 23 cases in grade III and 20 cases in grade IV. The tumor samples were subsequently classified into a lowgrade group (grade I-II) and high-grade group (grade III-IV). None of the patients received preoperative antitumor therapy. Each patient signed an informed consent form, and the study was approved by the Ethics Committee of China Medical University and the Review Board of the First Hospital of China Medical University and met the requirements of the Helsinki Declaration by the World Medical Association.

Cell culture. The human astrocytoma cell line U251 was purchased from the Cell Resource Center of Shanghai Institutes for Biological Sciences, the Chinese Academy of Science, and the cells were cultured in Dulbecco's modified Eagle's medium (DMEM, high-glucose) containing 10\% fetal bovine serum (FBS) (both from HyClone, Logan, UT, USA) at $37^{\circ} \mathrm{C}$ in $5 \%$ $\mathrm{CO}_{2}$ and $100 \%$ humidity. The medium was changed regularly, and the cells were treated with routine enzymatic digestion and passage.

Intracellular localization of nNav1.5 protein. U251 cells in the logarithmic growth phase were seeded onto 6-well plates (Corning, Corning, NY, USA) pre-set with coverslips. When the confluence of the adherent growing cells reached $50-60 \%$, the coverslips were taken out and fixed. The cells were then incubated with rabbit anti-human Nav1.5 polyclonal antibody (1:150; Abcam, Cambridge, UK) and mouse anti-human GFAP monoclonal antibody (1:100; Abcam) at room temperature overnight, and then incubated with DyLight 594-labeled goat anti-rabbit IgG (1:150; EarthOx, USA) and FITClabeled goat anti-mouse $\operatorname{IgG}(1: 150$; Jackson, USA) at room temperature for $2 \mathrm{~h}$. Cell nuclei were counterstained with 4,6-diamidino-2-phenylindole (DAPI; Invitrogen, Carlsbad, CA, USA). Results were visualized under a laser confocal microscope (Olympus FluoView FV300; Olympus Co., Tokyo, Japan).

Real-time quantitative RT-PCR. Total RNA was extracted from the tissues using the RNAiso Plus kit (Takara, Otsu, Shiga, Japan), and the RNA was reversely transcribed into cDNA according to the manufacturer's instructions (Takara). Gene-specific primers were designed and synthesized by Takara Inc. (Dalian, China), and the sequences are listed in Table I. Real-time RT-PCR was performed on an ABI PRISM 7000 Real-Time PCR System (Applied Biosystems, Foster City, CA, USA) using the SYBR Premix Ex Taq ${ }^{\mathrm{TM}}$ kit (Takara, Japan). The reaction conditions consisted of $95^{\circ} \mathrm{C}$ for 5 min followed by 40 cycles of $95^{\circ} \mathrm{C}$ for $1 \mathrm{~min}, 58^{\circ} \mathrm{C}$ for $1 \mathrm{~min}$ and $72^{\circ} \mathrm{C}$ for $1 \mathrm{~min}$. For each analysis, results were calculated using the $2^{-\Delta \Delta \mathrm{Ct}}$ method and normalized by the expression of $\beta$-actin. Reactions were performed in triplicate for each sample.

Immunohistochemistry. All tissue specimens were fixed with $4 \%$ paraformaldehyde to prepare the paraffin-embedded specimens in $4-\mu \mathrm{m}$ sections. Immunohistochemistry was performed according to the manufacturer's instructions for the Histostain-SP kit (Invitrogen). Rabbit anti-human polyclonal Nav1.5 antibody (1:250; Abcam) and biotinylated goat antirabbit IgG were used as the primary and secondary antibody, respectively. After counterstaining with 3,3'-diaminobenzidine (DAB), the sections were inspected under an optical microscope (BX40; Olympus Co.). For the control sections, the primary antibody was replaced by phosphate-buffered saline (PBS).

Western blot assay. Approximately $100 \mathrm{mg}$ of tissue was used for sodium dodecyl sulfate polyacrylamide gel electrophoresis (SDS/PAGE) and western blot analysis, as described in a previous study (13). After the protein concentration was determined using the BCA protein assay kit (R\&D Systems, Minneapolis, MN, USA) according to the manufacturer's instructions, $50 \mu \mathrm{g}$ of protein was resolved by SDS-PAGE, transferred onto polyvinylidene fluoride (PVDF) membranes (Roche, Indianapolis, IN, USA) by electroblotting, probed with specific primary antibodies, followed by secondary antibody conjugation and analyzed. The primary antibody was rabbit anti-human Nav1.5 polyclonal antibody (1:500; Abcam) and was detected using horseradish peroxidase-labeled goat anti- 
Table II. Primers of SCN5A/nNav1.5 siRNA.

\begin{tabular}{ll}
\hline Gene & \multicolumn{1}{c}{ Primer sequences } \\
\hline siRNA-1 (F) & 5'-GAGAUGACCUUCAAGAUCAdTdT-3' \\
siRNA-1 (R) & 5'-UGAUCUUGAAGGUCAUCUCdTdT-3' \\
siRNA-2 (F) & 5'-GAGUGAAGUUGGUGGUCAUdTdT-3' \\
siRNA-2 (R) & 5'-AUGACCACCAACUUCACUCdTdT-3' \\
siRNA-3 (F) & 5'-CAUUAUGCCUGCUGGUCUUdTdT-3' \\
siRNA-3 (R) & 5'-AAGACCAGCAGGCAUAAUGdTdT-3' \\
siRNA-NC (F) & 5'-UUCUCCGAACGUGUCACGUTT-3' \\
siRNA-NC (R) & 5'-ACGUGACACGUUCGGAGAATT-3' \\
\hline
\end{tabular}

nNav1.5, neonatal Nav1.5; siRNA, small interfering RNA.

rabbit secondary antibody (1:10,000; Santa Cruz, Santa Cruz, CA, USA). Immunoreactive protein bands were detected with an enhanced chemiluminescence reagent (ECL-Plus) and densitometrically quantitated according to the manufacturer's instructions (Amersham Pharmacia Biotech, Piscataway, NJ, USA).

Design and transfection of siRNA. The siRNA and siRNA-NC primers were designed and synthesized by Sigma-Aldrich Trading Co., Ltd. (Shanghai, China); sequences are presented in Table II. Well-growing U251 cells in the logarithmic growth phase were seeded at a density of $1 \times 10^{6}$ cells/well in 6-well plates (Corning) and cultured for $12 \mathrm{~h}$. The following groups were established for the experiment: the blank group (without any treatment), the control group (transfected with siRNANC), the siRNA-1 group (transfected with siRNA-1), the siRNA-2 group (transfected with siRNA-2) and the siRNA-3 group (transfected with siRNA-3). Transfection was carried out according to the instructions for the Lipofectamine ${ }^{\mathrm{TM}} 2000$ reagent (Invitrogen) as previously described (14).

$m R N A$ and protein determination following cell transfection. Transfection was followed by real-time quantitative RT-PCR and western blot assay to detect the nNav1.5 expression in the cells after 24 and $48 \mathrm{~h}$, respectively. The detailed procedures were the same as mentioned in the 'Real-time quantitative RT-PCR' and 'Western blot assay' sections.

MTT proliferation assay. After adjusting the cell density to $3 \times 10^{4} / \mathrm{ml}$, well-growing U251 cells in the logarithmic growth phase were seeded onto a 96 -well plate (Corning) at $100 \mu \mathrm{l} /$ well. Eight duplicate wells were established for each group. The cells were placed in a $37^{\circ} \mathrm{C}, 5 \% \mathrm{CO}_{2}$, saturated humidity incubator for conventional culture, and the transfection with medium change was carried out after $24 \mathrm{~h}$. The experimental groups were as follows: the blank group (without any treatment), the control group (transfected with siRNA-NC) and the siRNA group (transfected with siRNA-1). The transfection procedure was the same as above, and the cells continued to be routinely cultured after transfection. The assay was performed at 0 , 24, 48, 72 and $96 \mathrm{~h}$. For the assay, $10 \mu \mathrm{l}$ of CCK-8 reagent (Dojindo Molecular Technologies, Kumamoto, Japan) was added to each well, vortexed to mix and incubated at $37^{\circ} \mathrm{C}$ for $1 \mathrm{~h}$. The absorbance (OD) value at $450 \mathrm{~nm}$ for each well was measured with a microplate reader (Bio-Rad, Hercules, CA, USA). With time on the $\mathrm{x}$-axis and the OD value on the $y$-axis, the growth curve was plotted for each group of cells and the inhibition rate of cell proliferation was calculated.

Wound healing assay. U251 cells before and after transfection were subjected to a wound healing assay. Cells in the logarithmic growth phase were seeded onto 6-well plates (Corning) at a density of $1 \times 10^{6} /$ well. After culture for $24 \mathrm{~h}$, the medium was aspirated and discarded. A scratch in the shape of a straight line was created along the bottom of the well with a 10- $\mu \mathrm{l}$ pipette tip. The unattached cells were washed away with PBS, and the image was photographed for data recording. Ten evenly spaced points were selected at the edges of the scratch on each side, and the midline represented the edge of the scratch. The interval between the scratched cells was measured and recorded under an inverted microscope with a ruler $(0 \mathrm{~h})$. Changes after the 24 -h interval were recorded. The cell metastasis distance was calculated by subtracting the 24-h interval from the 0 -h interval.

Matrigel invasion assay. The Transwell chamber invasion assay kit (Corning) was used to detect changes in the cell invasiveness $24 \mathrm{~h}$ after transfection with the same experimental groups as in the proliferation assays. In short, Transwell inserts with an 8- $\mu \mathrm{m}$ pore size were coated with Matrigel (BD Biosciences, San Jose, CA, USA). The upper wells contained serum-free DMEM, and DMEM with 15\% FBS was used as an attractant for the lower wells. Six hours after transfection, the U251 cells were harvested and trypsinized into singlecell suspensions. The cell density was adjusted to $1 \times 10^{6} / \mathrm{ml}$, and $100 \mu \mathrm{l}$ of cells were seeded into each upper chamber. After $24 \mathrm{~h}$ of incubation, the non-invading cells in the upper chambers were gently wiped away, and the adherent cells on the lower side of each membrane were fixed and stained with trypan blue solution. Ten fields were randomly selected and counted under a microscope. Quantification of invasion was performed by counting the stained cells through the coated membranes. The cell invasion index percentage was calculated by dividing the number of cells in the lower chamber by the number of cells seeded in the upper chamber and then multiplying by $100 \%$.

Flow cytometric assay. Annexin V-FITC/PI (Annexin V-FITC/ PI kit; BD, San Diego, CA, USA) double staining was applied to detect apoptosis. U251 cells in the logarithmic growth phase were selected for the assay with the same experimental groupings as in the proliferation assay. Seventy-two hours after transfection, the cells from each sample were trypsinized and $1 \times 10^{4}$ cells were centrifuged and collected. Cells were re-suspended in $200 \mu 1$ of staining buffer and mixed with $10 \mu 1$ of Annexin V-FITC for 15 min before being filtered using a $200-\mu \mathrm{m}$ mesh. After adding $300 \mu \mathrm{l}$ of staining buffer and $5 \mu 1$ of propidium iodide (PI), flow cytometry (BD, San Jose, CA, USA) was performed to analyze the percentage of apoptotic cells.

Statistical analysis. Data were analyzed using one-way ANOVA followed by the Student's t-test using SPSS 18.0 statis- 

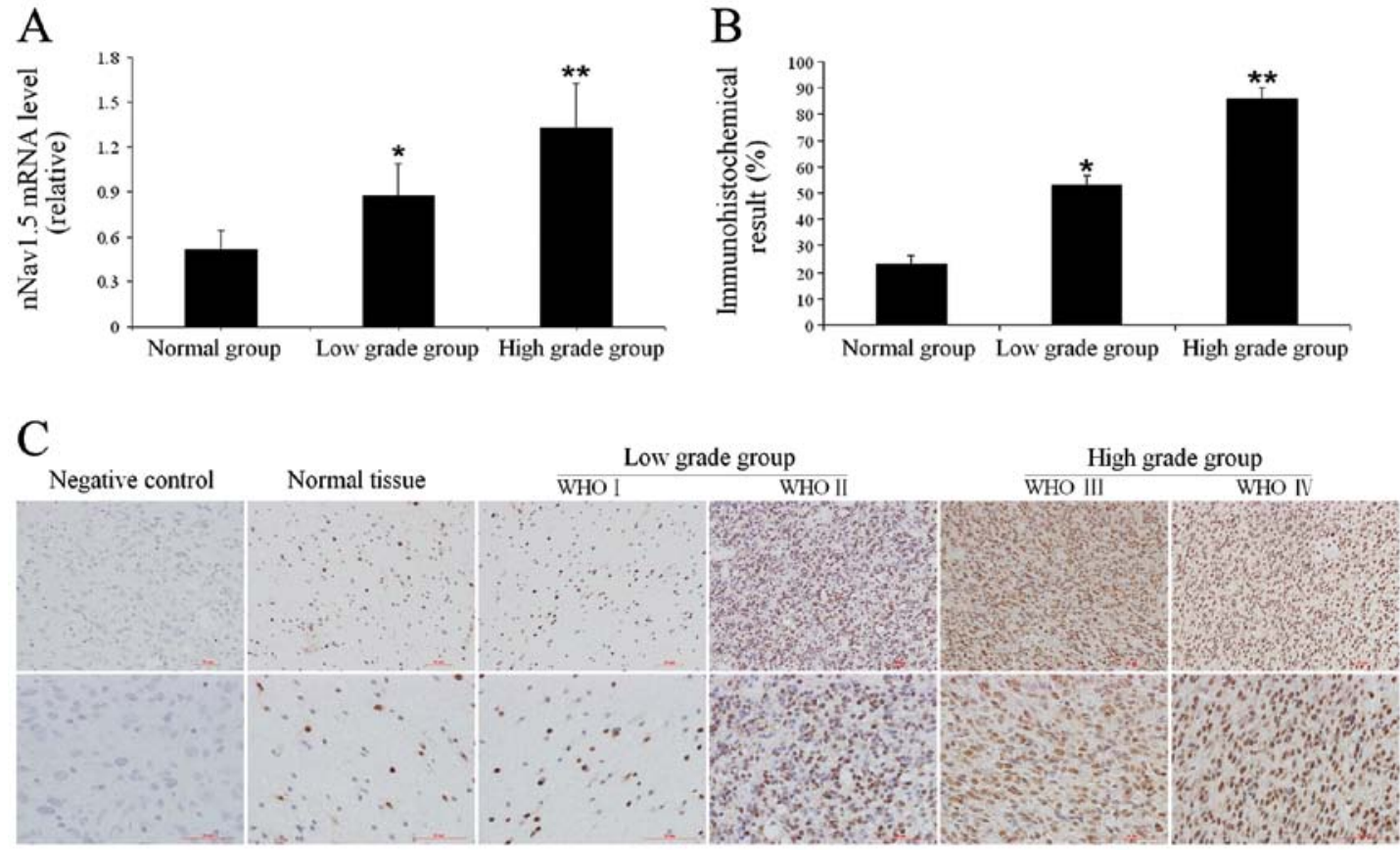

$\mathrm{D}$

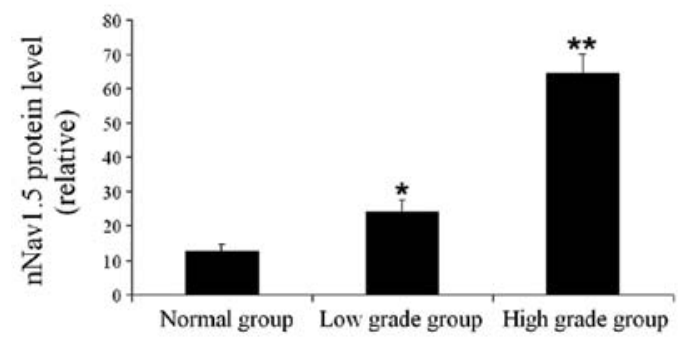

$\mathrm{E}$

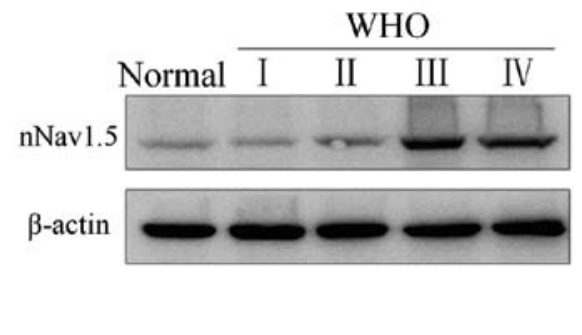

Figure 1. nNav1.5 expression in normal brain tissues and astrocytomas. (A) The relative expression levels of nNav1.5 mRNA in normal brain tissues and astrocytomas of different grades. (B) The positive expression rates of nNav1.5 in normal brain tissues and astrocytomas of different grades detected by immunohistochemistry. (C) Immunohistochemistry showed that in normal brain tissues and astrocytomas, nNav1.5 protein was expressed mainly in the nucleus, partially in the cytoplasm and cell membrane. (D) nNav1.5 protein expression in normal brain tissues and astrocytomas. (E) Bands of the western blot assay. $\left({ }^{*} \mathrm{P}<0.05\right.$ compared with the normal brain group with one-way ANOVA test and Student's t-test for multiple comparisons; ${ }^{* *} \mathrm{P}<0.05$ compared with the normal brain group and low-grade group with one-way ANOVA test and Student's t-test for multiple comparisons). nNav1.5, neonatal Nav1.5.

tical software (SPSS, Inc., Chicago, IL, USA). Comparisons were made between appropriate groups, and differences were considered statistically significant at the level of $\mathrm{P}<0.05$. Results are expressed as means \pm standard deviation.

\section{Results}

Expression of nNav1.5 in astrocytoma. Real-time quantitative RT-PCR confirmed nNav1.5 mRNA expression both in the astrocytoma and normal brain tissues. As shown in Fig. 1A, the relative levels of nNav1.5 mRNA in the low-grade group and high-grade group were $0.875 \pm 0.219$ and $1.327 \pm 0.303$, respectively, while that in the normal brain group was merely $0.509 \pm 0.131$. The immunohistochemistry and western blot analyses also confirmed nNav1.5 protein expression in both normal brain tissues and astrocytomas, while its expression in tumor tissues was significantly higher, with an increasing trend correlated with increasing pathological grade (Fig. 1B and D). The protein and mRNA expression levels were consistent. The nNav1.5 positive expression rates were determined by esti- mation of the number of nNav1.5-positive cells in the tissue specimens. The results of the immunohistochemistry showed that nNav1.5 protein was expressed mainly in the nucleus, with only a small amount of expression in the cytoplasm and cell membrane (Fig. 1C). The positive expression rates in normal brain tissues, low-grade astrocytomas, and highgrade astrocytomas were $23.0 \pm 3.4,52.9 \pm 3.9$ and $85.8 \pm 4.3 \%$, respectively, with statistically significant differences among the 3 groups (Fig. 1B). Western blotting showed that the relative expression levels of nNav1.5 protein in normal brain tissues, low-grade astrocytomas, and high-grade astrocytomas were $12.7 \pm 2.1,24.1 \pm 3.3$ and $64.3 \pm 5.9 \%$, respectively. The differences among the groups were statistically significant (Fig. 1D and E).

Localization of nNav1.5 protein in the U251 cells. As observed under confocal microscopy, nNav1.5 protein was expressed in the nucleus, cytoplasm and membrane (Fig. 2A) of U251 cells, which was consistent with the results of the immunohistochemistry assay (Fig. 1C). 

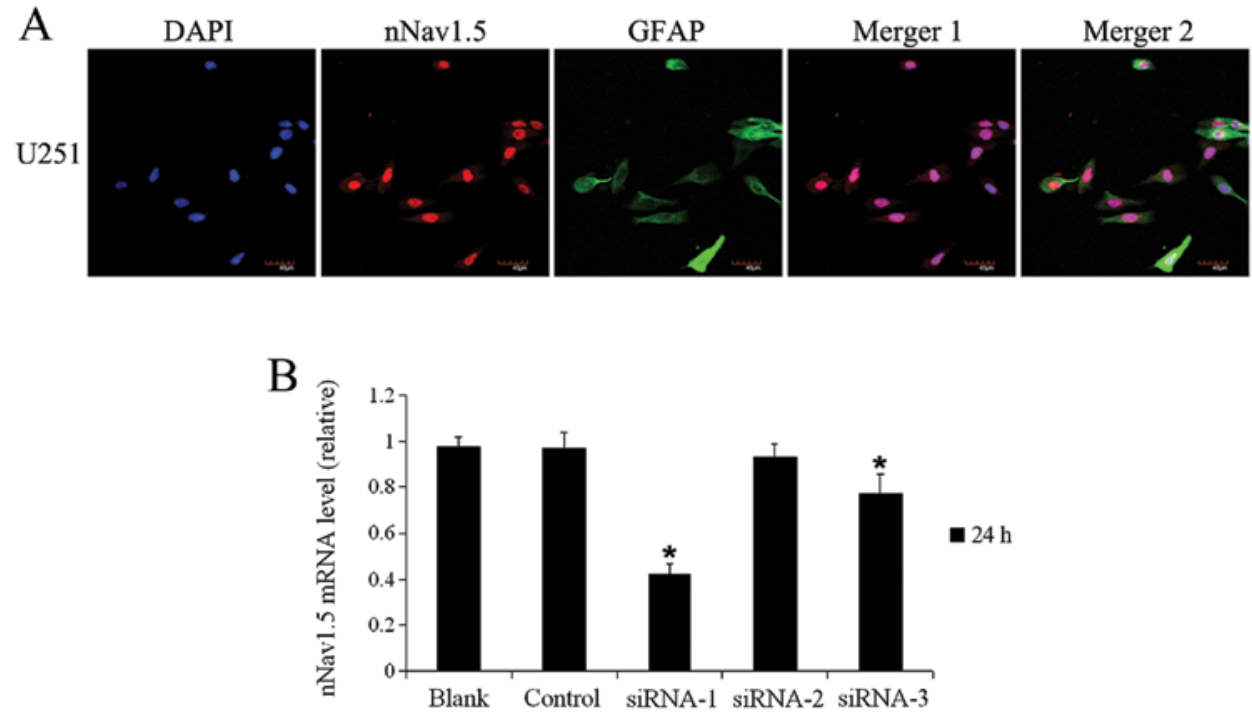

$\mathrm{C}$

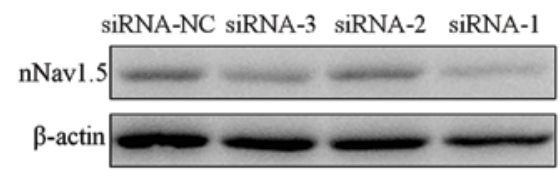

$\mathrm{D}$

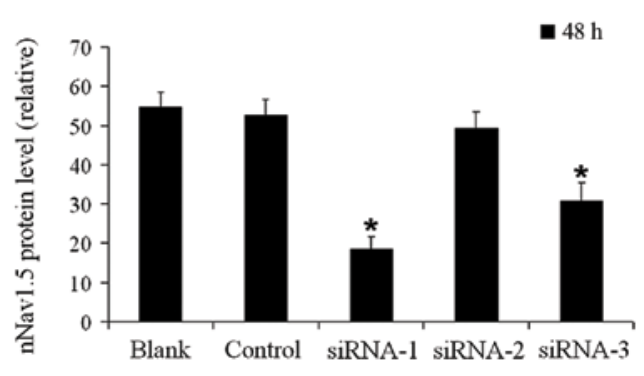

Figure 2. nNav1.5 siRNA reduces nNav1.5 expression in the U251 cells. (A) Immunofluorescence of nNav1.5 expression in cells under laser scanning confocal microscope. DAPI (blue), nNav1.5 (red) and GFAP (yellow) triple staining of human glioma U251 cells shows that the distribution of nNav1.5 is mainly located in the nucleus, and partially in the cytoplasm and cell membrane. (Merger 1, DAPI and nNav1.5; merger 2, DAPI, nNav1.5 and GFAP). (B) Relative nNav1.5 mRNA levels in cells $24 \mathrm{~h}$ after transfection with siRNA-NC or siRNA targeting nNav1.5. (C) Bands of the western blot assays after transfection. (D) Histogram of nNav1.5 protein expression after transfection. Values represent means \pm SD of 3 independent experiments $\left({ }^{*} \mathrm{P}<0.05\right.$ compared with the control group with one-way ANOVA test and Student's t-test for multiple comparisons). nNav1.5, neonatal Nav1.5; siRNA, small interfering RNA.

nNav1.5 siRNA significantly inhibits the expression of nNav1.5 in U251 cells. Twenty-four hours after siRNA transfection, the relative expression level of nNav1.5 mRNA was $0.978 \pm 0.043$ in the blank group, $0.968 \pm 0.071$ in the control group, $0.418 \pm 0.044$ in the siRNA-1 group, $0.930 \pm 0.054$ in the siRNA-2 group and $0.772 \pm 0.086$ in the siRNA-3 group, respectively (Fig. 2B). The transfection efficiency was the highest in the siRNA-1 group compared with the siRNA-2 and siRNA-3 groups. Compared with the blank and control groups, statistically significant differences were found in the siRNA-1 group and siRNA-3 group (Fig. 2B), with a reduced $n N a v 1.5$ mRNA expression level by 57.2 and $21.0 \%$, respectively. For the siRNA-2 group, the difference was not statistically significant (Fig. 2B). The difference between the control group and blank group was also not statistically significant (Fig. 2B).

To further verify the interference effect of the 3 synthesized siRNAs on nNav1.5 protein expression, the protein levels in the U251 cells were measured $48 \mathrm{~h}$ after transfection (Fig. 2C). As shown in Fig. 2D, in the siRNA-1 group the relative nNav1.5 protein level was $18.6 \pm 3.3 \%$, which was significantly lower than $49.2 \pm 4.2 \%$ in the siRNA-2 group and $30.9 \pm 4.5 \%$ in the siRNA-3 group. The expression level of $\mathrm{nNav1.5}$ protein in the $\mathrm{U} 251$ cells $48 \mathrm{~h}$ after transfection with

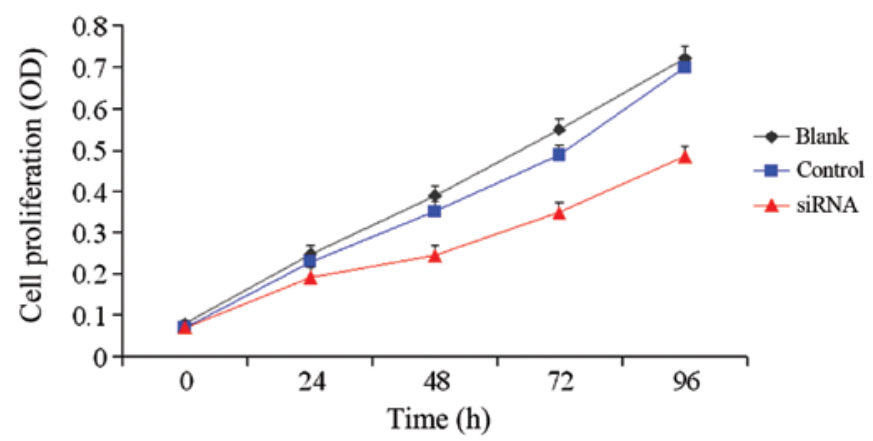

Figure 3. Growth curve of cell proliferation indicates that nNav1.5 siRNA had an inhibitive effect on U251 cells. Values represent means \pm SD of 3 independent experiments ( ${ }^{*} \mathrm{P}<0.05$ compared with the control group). nNav1.5, neonatal Nav1.5; siRNA, small interfering RNA.

siRNA-1 was consistent with the nNav1.5 mRNA expression level at $24 \mathrm{~h}$ (Fig. 2B and D). These data indicated that the nNav1.5 expression in U251 cells was efficiently inhibited $24 \mathrm{~h}$ after transfection with siRNA, and siRNA-1 was selected for the subsequent cellular experiments. 

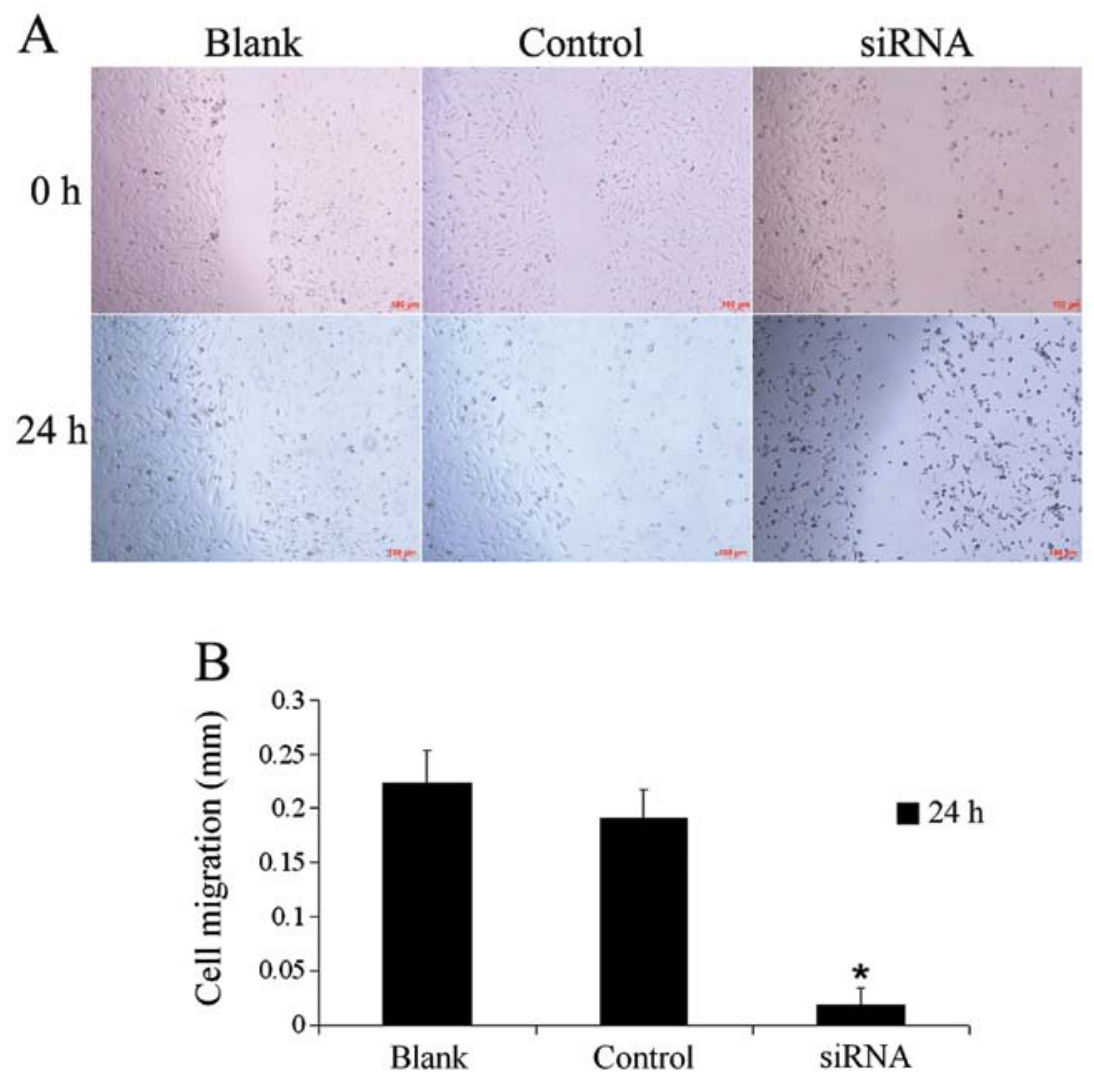

Figure 4. nNav1.5 siRNA reduces VGSC-dependent cell migration in U251 cells. (A) Wound healing assay after transfection with siRNA-NC or siRNA targeting nNav1.5. (B) Cell migration distance $(\mathrm{mm})$ over $24 \mathrm{~h}$ after transfection with siRNA-NC or siRNA targeting nNav1.5. Values represent means \pm SD of 3 independent experiments ("P $<0.05$ compared with the control group). nNav1.5, neonatal Nav1.5; siRNA, small interfering RNA; VGSC, voltage-gated sodium channel.

nNav1.5 siRNA inhibits the in vitro proliferation of $U 251$ cells. In the MTT proliferation assay, the CCK-8 assay was utilized to detect changes in the in vitro proliferation of U251 cells after transfection. As shown in Fig. 3, the OD values of the siRNA group at each time point were statistically significantly different when compared with those of the control group and blank group. The inhibition rates of siRNA-1 were $22.7 \%$ at $24 \mathrm{~h}, 36.9 \%$ at $48 \mathrm{~h}, 36.4 \%$ at $72 \mathrm{~h}$ and $32.7 \%$ at $96 \mathrm{~h}$, respectively. The inhibition of cell proliferation peaked at $48 \mathrm{~h}$, and the interference effect of the siRNA weakened over time. These data suggest that the proliferation of the U251 cells was significantly inhibited by siRNA interference of SCN5A/ nNav1.5 expression.

nNav1.5 siRNA reduces the migratory ability of U251 cells. The wound healing assay (Fig. 4A) revealed that the distance of migration of the cells in the siRNA group at $24 \mathrm{~h}(0.019 \pm 0.015 \mathrm{~mm})$ was significantly shorter than the distances in the blank group $(0.223 \pm 0.031 \mathrm{~mm})$ and control group $(0.190 \pm 0.027 \mathrm{~mm})$, and the differences were statistically significant (Fig. 4B). Compared with the control group, the migration distance in the siRNA group was decreased by $91.6 \%$.

nNav1.5 siRNA inhibits the invasiveness of U251 cells. The Matrigel invasion assay (Fig. 5) showed that the invasion index of the U251 cells in the siRNA group $(2.99 \pm 0.15 \%)$ was significantly lower than the indices in the blank group $(6.77 \pm 0.26 \%)$ and control group $(6.60 \pm 0.33 \%)$. The cell invasiveness in the siRNA group was decreased by $55.83 \%$ compared with the invasiveness of the blank group.

nNav1.5 siRNA promotes the apoptosis of U251 cells. U251 cells were harvested $72 \mathrm{~h}$ after transfection to measure the apoptotic rates. As shown in Fig. 6, the apoptotic rates were $21.66 \pm 1.55,5.71 \pm 0.42$ and $6.54 \pm 0.86 \%$ in the siRNA, blank and control groups, respectively. The apoptotic rate was significantly lower than that in either the blank group or the control group.

\section{Discussion}

Astrocytoma is the most common primary CNS malignant tumor and is frequently infiltrative and recurs easily, with poor patient prognosis (15). Treatment for astrocytoma involves combined therapies including surgery, chemotherapy, radiotherapy and immunotherapy. However, the outcome of astrocytoma is still dismal. Currently, progress in immunotherapy holds hope for the treatment of astrocytoma and has become a promising option (16-18). Thus, it is necessary to search for new therapeutic targets for astrocytoma.

Studies have found that various ion channels, including $\mathrm{Na}^{+}, \mathrm{K}^{+}, \mathrm{Cl}^{-}$and $\mathrm{Ca}^{2+}$ are widely distributed in the membrane structures of astrocytoma cells. These channels not only play important roles in various physiological activities such as growth and metabolism in glial cells (19), but also in cell cycle 

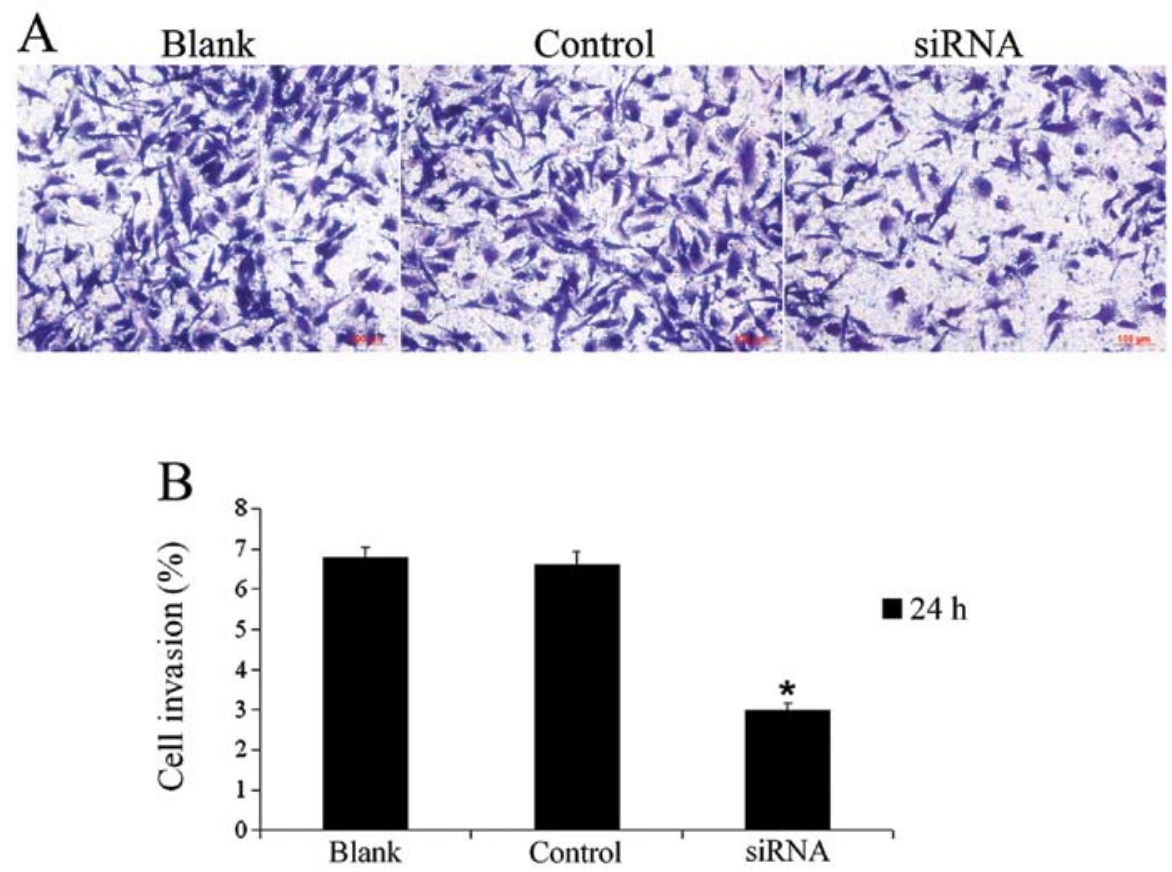

Figure 5. nNav1.5 siRNA reduces VGSC-dependent cell invasiveness. (A) Matrigel invasion assay after transfection with siRNA-NC or siRNA targeting nNav1.5. (B) Quantitative comparison of migrated cells. Values represent means \pm SD of 3 independent experiments ("P $<0.05$ compared with the control group). nNav1.5, neonatal Nav1.5; siRNA, small interfering RNA; VGSC, voltage-gated sodium channel.
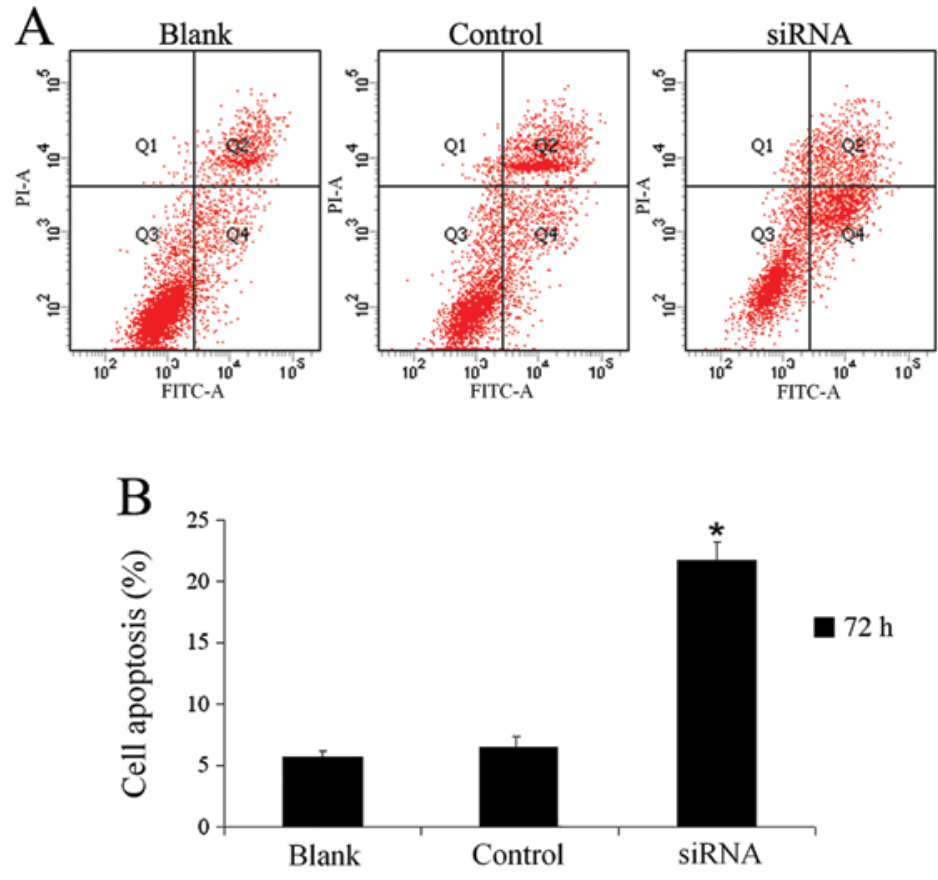

Figure 6. nNav1.5 siRNA promotes cell apoptosis. (A) Flow cytometry after transfection with siRNA-NC or siRNA targeting nNav1.5. (B) Seventy-two hours after transfection with siRNA-NC or siRNA targeting nNav1.5, the cell apoptotic rate was significantly increased. Values represent means \pm SD of 3 independent experiments (" $\mathrm{P}<0.05$ compared with the control group). nNav1.5, neonatal Nav1.5; siRNA, small interfering RNA.

regulation, proliferation, metastasis, invasion and apoptosis of astrocytoma cells $(8,19-25)$. VGSCs are mainly expressed in tumors of epithelial origin, and can participate in the biological behaviors of tumor cells, including direct spread, lateral movement, galvanotaxis, invasion and the secretory activity of the membrane $(8,26,27)$. In the present study, we proposed that various VGSC subtypes may be related to the occurrence and development of astrocytoma, and we carried out a series of assays to confirm this hypothesis. Our preliminary findings confirmed that Nav1.5 is widely distributed in the CNS. Therefore, we first cloned the complete sequence of the Nav1.5 gene expressed in human cortical neurons and 
identified its function (8). The results showed that the SCN5A gene which encodes Nav1.5 in brain tissue is different than that in human cardiomyocytes, and they belong to two different subtypes. The former is a neonatal isoform $(6,7,9,28$ 31), namely nNav1.5 (neonatal Nav1.5), while the latter is a mature isoform. Our and various other studies suggest that the Nav1.5 subtype of VGSCs encoded by the SCN5A gene could potentially serve as a marker of tumor metastasis and as a therapeutic target (8,10-12,26,30-36). Recent studies have found that the functions of VGSCs may vary with changes in mRNA splicing (2). Exon 6, located in the DI: S3 segment plays an important role in regulating development. The 5 ' neonatal isoform can be expressed at birth, while the $3^{\prime}$ mature isoform is expressed later in development (37). Our study confirmed that the nNav1.5 $\mathrm{Na}^{+}$channel is widely expressed in rat CNS $(9,28)$. However, it is worth noting that the average expression level of nNav1.5 showed a downward trend as the age of the rats increased $(7,9,27,30)$, indicating that it is mainly expressed in immature neurons or glial cells, further suggesting that the expression of nNav1.5 may be the result of the re-expression of an embryonic gene or oncogene.

As the power pump of cellular activity, $\mathrm{Na}^{+}$channels play an important role in maintaining the physiological activities of cells (38-40). Several studies found that the Nav1.5 protein encoded by the SCN5A gene was mainly expressed in the cell membrane and cytoplasm of other tumors $(32,41)$. In the present study, the expression levels of nNav1.5 mRNA and protein in astrocytoma and normal brain tissues were first assessed in detail. Furthermore, cytological immunofluorescence studies found that $\mathrm{nNav1.5}$ protein was expressed in the nucleus, membrane and cytoplasm of U251 cells, while the immunohistochemistry also confirmed the expression of nNav1.5 in the various locations described above. However, its expression level in astrocytoma cells was significantly higher than that in brain tissues. With reduced differentiation of the astrocytoma nucleus, nNav1.5 expression became more significant. The levels of expression of SCN5A/nNav1.5 in high-grade astrocytoma with a low degree of differentiation and in the low-grade astrocytoma with a high degree of differentiation were also significantly different, suggesting that the expression level of nNav1.5 is positively correlated with the degree of malignancy. The expression of nNav1.5 protein in cell nuclei suggested that the VGSC isoform nNav1.5 may play an important role in the processes of tumor cell division and malignant proliferation. These data revealed upregulation of nNav1.5 expression in astrocytoma and a positive correlation with pathological grade. These findings further suggest that nNav1.5 encoded by the SCN5A gene may be the re-expression of an embryonic gene or oncogene that could be a novel diagnostic or therapeutic target for astrocytoma. Moreover, no study concerning the regulation of nNav1.5 in the occurrence and development of astrocytoma has been reported to date. To further understand the specific role of nNav1.5 in the occurrence and development of astrocytoma, RNAi technology was used to knock down the expression of the SCN5A/nNav1.5 gene in U251 cells. Effective transfection of the cells and significant inhibition of nNav1.5 mRNA and protein expression were observed, indicating that the RNAi interference sequence specifically inhibited the expression of nNav1.5. After RNAi knockdown, suppressed expression of nNav1.5 inhibited proliferation, reduced migration and invasiveness, and induced a higher apoptosis rate compared with the blank and negative control groups, indicating that the downregulation of nNav1.5 expression significantly inhibited the proliferation, migration and invasion of U251 cells and played a significant role in promoting tumor cell apoptosis. Therefore, nNav1.5 has the potential to be a novel molecular therapeutic target for astrocytoma, with great significance in cancer prognosis and clinical treatment, although the mechanism remains unclear. According to a recent report, VGSCs most likely enhance the invasion and metastatic behavior in tumor cells through three types of regulatory mechanisms: $\mathrm{pH}$ adjustment, regulation of gene expression and intracellular $\mathrm{Ca}^{2+}$ regulation (42). It has been reported that $\mathrm{Na}^{+}$influx can promote the proliferation of cancer cells. If a spontaneous $\mathrm{Na}^{+}$ influx occurs in the early proliferative phase, controlling the external concentration of $\mathrm{Na}^{+}$could effectively inhibit cell proliferation (43). Another study revealed that the Nav1.5 and Nav1.7 subtypes are found in endothelial cells of the human umbilical vein, and the inhibition of Nav1.5 expression in these cells by RNAi inhibited cell proliferation (44). However, in the prostate cancer Mat-Lylu cell line and breast cancer MDA-MB-231 cells with high metastatic potential, VGSC activity was not related to cell proliferation $(11,45)$. These differences may be due to differences between the cell types.

In summary, the nNav1.5 encoded by the SCN5A gene was functionally highly expressed in human brain astrocytoma, and the expression levels were correlated with the degree of malignancy. Following downregulation of its expression level, the proliferation, migration and invasion of the astrocytoma cells were significantly inhibited, and their apoptotic rate was markedly increased. However, the underlying mechanisms are still unclear and further investigation is needed. To our knowledge, this is the first study to detect the expression and function of nNav1.5 in astrocytoma.

\section{Acknowledgements}

This study was supported by the National Natural Science Foundation of China (no. 31100770 to J.W.) and the Liaoning Provincial Natural Science Foundation of China (no. 2013021075 to B.Q.). We thank all the staff members of the Neurosurgery Department of the First Hospital of China Medical University for their technical help.

\section{References}

1. Binello E and Germano IM: Targeting glioma stem cells: a novel framework for brain tumors. Cancer Sci 102: 1958-1966, 2011.

2. Diss JK, Fraser SP and Djamgoz MB: Voltage-gated $\mathrm{Na}^{+}$channels: multiplicity of expression, plasticity, functional implications and pathophysiological aspects. Eur Biophys J 33: 180-193, 2004.

3. Monk M and Holding C: Human embryonic genes re-expressed in cancer cells. Oncogene 20: 8085-8091, 2001.

4. Kunzelmann K: Ion channels and cancer. J Membr Biol 205 : 159-173, 2005.

5. McFerrin MB and Sontheimer H: A role for ion channels in glioma cell invasion. Neuron Glia Biol 2: 39-49, 2006.

6. Brackenbury WJ, Djamgoz MB and Isom LL: An emerging role for voltage-gated $\mathrm{Na}^{+}$channels in cellular migration: regulation of central nervous system development and potentiation of invasive cancers. Neuroscientist 14: 571-583, 2008.

7. Wang J, Ou SW, Wang YJ, et al: New variants of Nav1.5/SCN5A encode $\mathrm{Na}^{+}$channels in the brain. J Neurogenet 22: 57-75, 2008. 
8. Ou SW, Kameyama A, Hao LY, et al: Tetrodotoxin-resistant $\mathrm{Na}^{+}$channels in human neuroblastoma cells are encoded by new variants of Nav1.5/SCN5A. Eur J Neurosci 22: 793-801, 2005.

9. Wang J, Ou SW, Wang YJ, Kameyama M, Kameyama A and Zong ZH: Analysis of four novel variants of Nav1.5/SCN5A cloned from the brain. Neurosci Res 64: 339-347, 2009.

10. Fraser SP, Diss JK, Lloyd LJ, et al: T-lymphocyte invasiveness: control by voltage-gated $\mathrm{Na}^{+}$channel activity. FEBS Lett 569: 191-194, 2004.

11. Fraser SP, Diss JK, Chioni AM, et al: Voltage-gated sodium channel expression and potentiation of human breast cancer metastasis. Clin Cancer Res 11: 5381-5389, 2005.

12. Brackenbury WJ, Chioni AM, Diss JK and Djamgoz MB: The neonatal splice variant of Nav1.5 potentiates in vitro invasive behaviour of MDA-MB-231 human breast cancer cells. Breast Cancer Res Treat 101: 149-160, 2007.

13. Qiu B, Sun X, Zhang D, Wang Y, Tao J and Ou S: TRAIL and paclitaxel synergize to kill U87 cells and U87-derived stem-like cells in vitro. Int J Mol Sci 13: 9142-9156, 2012.

14. Yan X, Liang H, Deng T, et al: The identification of novel targets of miR-16 and characterization of their biological functions in cancer cells. Mol Cancer 12: 92, 2013

15. Han G, Zhao W, Wang L, et al: Leptin enhances the invasive ability of glioma stem-like cells depending on leptin receptor expression. Brain Res 1543: 1-8, 2014.

16. Murphy KA, Erickson JR, Johnson CS, et al: $\mathrm{CD}^{+} \mathrm{T}$ cell-independent tumor regression induced by Fc-OX40L and therapeutic vaccination in a mouse model of glioma. J Immunol 192: 224-233, 2014.

17. Ning J, Wakimoto $H$ and Rabkin SD: Immunovirotherapy for glioblastoma. Cell Cycle 13: 175-176, 2014.

18. van Gool S: Immunotherapy for high-grade glioma: how to go beyond Phase I/II clinical trials. Immunotherapy 5: 1043-1046, 2013.

19. Kawaguchi A, Asano H, Matsushima K, Wada T, Yoshida S and Ichida S: Enhancement of sodium current in NG108-15 cells during neural differentiation is mainly due to an increase in $\mathrm{Na}_{\mathrm{v}} 1.7$ expression. Neurochem Res 32: 1469-1475, 2007.

20. Ross SB, Fuller CM, Bubien JK and Benos DJ: Amiloridesensitive $\mathrm{Na}^{+}$channels contribute to regulatory volume increases in human glioma cells. Am J Physiol Cell Physiol 293: C1181-C1185, 2007.

21. Weiss RE and Sidell N: Sodium currents during differentiation in a human neuroblastoma cell line. J Gen Physiol 97: 521-539, 1991.

22. Olsen ML, Schade S, Lyons SA, Amaral MD and Sontheimer H: Expression of voltage-gated chloride channels in human glioma cells. J Neurosci 23: 5572-5582,2003.

23. Ducret T, Vacher AM and Vacher P: Voltage-dependent ionic conductances in the human malignant astrocytoma cell line U87-MG. Mol Membr Biol 20: 329-343, 2003.

24. Vila-Carriles WH, Kovacs GG, Jovov B, et al: Surface expression of ASIC2 inhibits the amiloride-sensitive current and migration of glioma cells. J Biol Chem 281: 19220-19232, 2006.

25. Wang WX and Ji YH: Scorpion venom induces glioma cell apoptosis in vivo and inhibits glioma tumor growth in vitro. J Neurooncol 73: 1-7, 2005.

26. Onganer PU and Djamgoz MB: Small-cell lung cancer (human) potentiation of endocytic membrane activity by voltage-gated $\mathrm{Na}^{+}$channel expression in vitro. J Membr Biol 204: 67-75, 2005.

27. Mycielska ME, Fraser SP, Szatkowski M and Djamgoz MB: Contribution of functional voltage-gated $\mathrm{Na}^{+}$channel expression to cell behaviors involved in the metastatic cascade in rat prostate cancer: II. Secretory membrane activity. J Cell Physiol 195 461-469, 2003
28. Ren CT, Li DM, Ou SW, et al: Cloning and expression of the two new variants of Nav1.5/SCN5A in rat brain. Mol Cell Biochem 365: 139-148, 2012.

29. Catterall WA: From ionic currents to molecular mechanisms: the structure and function of voltage-gated sodium channels. Neuron 26: 13-25, 2000.

30. Roger S, Besson P and Le Guennec JY: Involvement of a novel fast inward sodium current in the invasion capacity of a breast cancer cell line. Biochim Biophys Acta 1616: 107-111, 2003.

31. Roger S, Rollin J, Barascu A, et al: Voltage-gated sodium channels potentiate the invasive capacities of human non-smallcell lung cancer cell lines. Int J Biochem Cell Biol 39: 774-786, 2007.

32. House CD, Vaske CJ, Schwartz AM, et al: Voltage-gated $\mathrm{Na}^{+}$ channel SCN5A is a key regulator of a gene transcriptional network that controls colon cancer invasion. Cancer Res 70: 6957-6967, 2010

33. Gao R, Shen Y, Cai J, Lei M and Wang Z: Expression of voltagegated sodium channel $\alpha$ subunit in human ovarian cancer. Oncol Rep 23: 1293-1299, 2010.

34. Diaz D, Delgadillo DM, Hernández-Gallegos E, et al: Functional expression of voltage-gated sodium channels in primary cultures of human cervical cancer. J Cell Physiol 210: 469-478, 2007.

35. Diss JK, Stewart D, Pani F, et al: A potential novel marker for human prostate cancer: voltage-gated sodium channel expression in vivo. Prostate Cancer Prostatic Dis 8: 266-273, 2005.

36. Yang M, Kozminski DJ, Wold LA, et al: Therapeutic potential for phenytoin: targeting $\mathrm{Na}_{\mathrm{v}} 1.5$ sodium channels to reduce migration and invasion in metastatic breast cancer. Breast Cancer Res Treat 134: 603-615, 2012.

37. Onkal R, Mattis JH, Fraser SP, et al: Alternative splicing of Nav1.5: an electrophysiological comparison of 'neonatal' and 'adult' isoforms and critical involvement of a lysine residue. J Cell Physiol 216: 716-726, 2008.

38. Aman TK, Grieco-Calub TM, Chen C, et al: Regulation of persistent $\mathrm{Na}$ current by interactions between $\beta$ subunits of voltage-gated Na channels. J Neurosci 29: 2027-2042, 2009.

39. Leterrier C, Brachet A, Fache MP and Dargent B: Voltage-gated sodium channel organization in neurons: protein interactions and trafficking pathways. Neurosci Lett 486: 92-100, 2010.

40. Corry B and Thomas M: Mechanism of ion permeation and selectivity in a voltage gated sodium channel. J Am Chem Soc 134: 1840-1846, 2012.

41. Chioni AM, Shao D, Grose R and Djamgoz MB: Protein kinase $\mathrm{A}$ and regulation of neonatal Nav1.5 expression in human breast cancer cells: activity-dependent positive feedback and cellular migration. Int J Biochem Cell Biol 42: 346-358, 2010.

42. Brackenbury WJ: Voltage-gated sodium channels and metastatic disease. Channels 6: 352-361, 2012.

43. Abdul M and Hoosein N: Voltage-gated potassium ion channels in colon cancer. Oncol Rep 9: 961-964, 2002.

44. Andrikopoulos P, Fraser SP, Patterson L, et al: Angiogenic functions of voltage-gated $\mathrm{Na}^{+}$channels in human endothelial cells: modulation of vascular endothelial growth factor (VEGF) signaling. J Biol Chem 286: 16846-16860, 2011.

45. Fraser SP, Grimes JA and Djamgoz MB: Effects of voltage-gated ion channel modulators on rat prostatic cancer cell proliferation: comparison of strongly and weakly metastatic cell lines. Prostate 44: 61-76, 2000. 